\title{
Desempenhos do Sensoriamento Espectral via Periodograma Ponderado sob Ruído não Uniforme e Canais Seletivos e com Sombreamento
}

\author{
Lucas dos Santos Costa, Wesley Reis da Silva e Rausley Adriano Amaral de Souza
}

\begin{abstract}
Resumo-O algoritmo de cancelamento cooperativo por divisão da densidade espectral de potência com dobramento circular (circular folding cooperative power spectral density split cancellation, CFCPSC) é um algoritmo baseado em periodogramas projetado para detectar sinais primários, via rádios cognitivos (cognitive radios, CRs), no sensoriamento espectral cooperativo centralizado com fusão de dados. Suas principais vantagens são: i) baixa complexidade de implementação e robustez em cenários sob ii) ruído dinâmico e iii) ruído não uniforme. Além disso, o CFCPSC mostrou-se efetivo em cenários sob iv) canais seletivos em frequência e/ou v) com sombreamento correlacionado e vi) ruído impulsivo. $\mathrm{O}$ algoritmo weighted CFCPSC, ou WCFCPSC, é a nova versão ponderada do CFCPSC com maior poder estatístico de detecção, contudo ainda não analisado nos cenários supracitados. Portanto, este artigo avalia os desempenhos do WCFCPSC nos cenários iii, iv e $v$ e os resultados mostram que seu maior poder estatístico se mantem também nestes cenários.
\end{abstract}

Palavras-Chave-sensoriamento espectral, rádios cognitivos, sombreamento correlacionado, canais seletivos em frequência, CFCPSC, WCFCPSC.

Abstract-The circular folding cooperative power spectral density split cancellation (CFCPSC) is a periodogram-based algorithm developed to detect primary signals, via cognitive radios (CRs), in centralized cooperative spectrum sensing with data fusion. Its main advantages are i) low implementation complexity and robustness in scenarios of ii) dynamical noise and iii) nonuniform noise. Besides, the CFCPSC showed to be effective in scenarios under iv) frequency-selective channels, combined or not with $v$ ) correlated shadowed-fading channels and vi) impulsive noise. The algorithm weighted CFCPSC, or WCFCPSC, is a new, weighted, version of the CFCPSC with larger statistical detection power, but not analyzed under the above-mentioned scenarios yet. Therefore, this paper analyses the WCFCPSC performances under the Scenarios iii, iv and $v$ and results show that its larger statistical power is maintained in these scenarios.

Keywords-spectrum sensing, cognitive radios, correlated shadowing, frequency-selective channels, CFCPSC, WCFCPSC.

\section{INTRODUÇÃO}

A expansão da demanda por novos serviços de comunicação é a principal razão para o problema da escassez espectral. Por isso, tal problema torna-se ainda mais evidente com os

Lucas dos Santos Costa, Wesley R. da Silva e Rausley A. A. de Souza, Instituto Nacional de Telecomunicações (Inatel), Santa Rita do Sapucaí, MG, e-mail: lucass@inatel.br, wesley.reis@mtel.inatel.br, rausley@inatel.br. Este trabalho foi parcialmente financiado Fapemig e pela RNP, com recursos do MCTIC, processo No 01245.010604/2020-14, sob o projeto Sistemas de Comunicações Móveis de $6^{\mathrm{a}}$ Geração (6G) do Centro de Referência em Radiocomunicações (CRR) do Instituto Nacional de Telecomunicações - Inatel, Brasil. adventos da quinta geração (5G) e da sexta geração (6G) dos sistemas de comunicação e a Internet das coisas (Internet of things, IoT), pois preveem coexistência entre um enorme número de dispositivos transceptores [1], bem como novas aplicações [2]. Contudo, pesquisas recentes mostram que o espectro de frequências se encontra contraditoriamente subutilizado [3], já que faixas ociosas podem ser frequentemente encontradas em determinadas localizações geográficas e determinados períodos de tempo. A ineficiência do uso do espectro se deve especialmente à política de acesso fixo em vigor, que concede direito de uso exclusivo a usuários contratantes, também chamados de usuários primários (primary users, PUs), durante o período de tempo contratado.

Percebeu-se que uma mudança da atual política de acesso fixo para uma política de acesso dinâmico poderia reduzir conjuntamente a ineficiência no uso do espectro e a escassez espectral. Nessa nova política de acesso, o espectro, outrora exclusivo para PUs, tornar-se-ia compartilhado oportunisticamente com usuários não contratantes, ou usuários secundários (secondary users, SUs), desde que canais primários ociosos pudessem ser detectados. Logo, seria imperativo que SUs fossem capazes de detectar oportunidades de acesso aos canais primários. Nesse contexto surgiu o conceito de sensoriamento espectral baseado em rádio cognitivo (cognitive radio, $\mathrm{CR}$ ). Um CR é então um dispositivo equipado com uma capacidade de cognição tal que o capacita a observar e inferir sobre estado de ocupação de um canal via sensoriamento dos sinais emitidos pelos PUs.

No sensoriamento espectral cooperativo centralizado com fusão de dados, CRs coletam informações de sensoriamento de um canal alvo e as enviam, via canal de controle, a um centro de fusão (fusion center, FC) para combinação e decisão sobre o estado de ocupação desse canal. A combinação é feita por meio de uma estatística de teste, e então uma decisão cooperativa mais acurada, devido ao processo de cooperação, sobre a ocupação do canal é tomada. Algumas estatísticas bem conhecidas são aquelas baseadas no detector de energia (energy detection, ED) [4] do sinal recebido, nos autovalores da matriz de covariância amostral do sinal recebido [5], e na estimativa da densidade espectral de potência (power spectral density, PSD) do sinal recebido [5].

As estatísticas de teste podem ser divididas em semicegas ou cegas. As cegas possuem a vantagem de serem computadas sem conhecimentos a priori sobre quaisquer características do sinal PU ou ruído, como no teste de razão de máxima verossimilhança generalizado (generalized likelihood ratio test, 
GLRT) [4] e no algoritmo de cancelamento cooperativo por divisão da densidade espectral de potência com dobramento circular (circular folding cooperative power spectral density split cancellation, CFCPSC) [5]. O CFCPSC ainda conta com as vantagens de possuir: i) baixa complexidade de implementação e robustez em cenários sob ii) ruído dinâmico (quando os SUs operam sob potência de ruído variante no tempo), iii) ruído não uniforme (quando cada SU opera sob uma potência média de ruído distinta), iv) canais seletivos em frequência e/ou com v) sombreamento correlacionado e vi) ruído impulsivo [5], [6].

Recentemente, foi proposta uma versão ponderada do algoritmo CFCPSC em [7]. Esta versão foi chamada (weighted CFCPSC), i.e. WCFCPSC, e resultou em um aumento significativo do poder estatístico de detecção do CFCPSC. Porém, os desempenhos do WCFCPSC não foram analisados em nenhum dos cenários supracitados. Portanto, neste artigo avalia-se os desempenhos do WCFCPSC em cenários de ruído não uniforme e sob canais seletivos em frequência e com sombreamento correlacionado. Seus desempenhos são comparados com os desempenhos obtidos pelo algoritmo original CFCPSC e pelo GLRT, que em alguns cenários superou o CFCPSC em [5]. Os resultados revelam que o WCFCPSC manteve seu maior poder estatístico em relação ao CFCPSC e inclusive, de forma destacada em alguns casos, superou o GLRT nos cenários que o CFCPSC obteve desempenhos inferiores em [5].

$\mathrm{O}$ restante deste artigo se encontra organizado da seguinte forma. A Seção II define a decisão de ocupação dos canais PUs no sensoriamento espectral como um teste binário de hipóteses, bem como apresenta o modelo do sistema, incluindo o modelo de canal de sensoriamento e os algoritmos GLRT, CFCPSC e WCFCPSC. A Seção III mostra os resultados obtidos e, por fim, as conclusões são apresentadas na Seção IV.

\section{Sensoriamento Espectral e Modelo do Sistema}

No sensoriamento espectral, a decisão sobre o estado de ocupação de um canal PU sob sensoriamento por um ou mais CRs, ou SUs, pode ser modelada como um teste binário de hipóteses, $\mathcal{H}_{0}$ e $\mathcal{H}_{1}$, sendo $\mathcal{H}_{0}$ e $\mathcal{H}_{1}$ as hipóteses desse canal estar livre ou ocupado, respectivamente. O desempenho dessa decisão é comumente mensurado pela probabilidade de falso alarme, $P_{\text {fa }}=\operatorname{Pr}\left\{\right.$ decisão $\left.=\mathcal{H}_{1} \mid \mathcal{H}_{0}\right\}=\operatorname{Pr}\left\{T>\gamma \mid \mathcal{H}_{0}\right\}$, e pela probabilidade de detecção, $P_{\mathrm{d}}=\operatorname{Pr}\left\{\right.$ decisão $\left.=\mathcal{H}_{1} \mid \mathcal{H}_{1}\right\}=$ $\operatorname{Pr}\left\{T>\gamma \mid \mathcal{H}_{1}\right\}$. $P_{\text {fa }}$ é a probabilidade de haver uma decisão em favor de $\mathcal{H}_{1}$, dado que o canal está sob hipótese $\mathcal{H}_{0}$ (livre), o que é equivalente à hipótese da estatística de teste $T$ ser maior que o limiar de decisão predefinido $\gamma$, ou seja $T>\gamma$, dado $\mathcal{H}_{0}$. Já $P_{\mathrm{d}}$ é a probabilidade de haver uma decisão em favor de $\mathcal{H}_{1}$, dado que o canal está de fato sob hipótese $\mathcal{H}_{1}$ (ocupado), o que é equivalente à possibilidade de $T>\gamma$, dado $\mathcal{H}_{1}$. As análises de desempenho são geralmente realizadas graficamente por meio da curva característica de operação do receptor (receiver operating characteristic, ROC), que mapeia $P_{\mathrm{fa}}$ e $P_{\mathrm{d}}$ com a variação do limiar de decisão $\gamma$, bem como por meio da área abaixo da curva ROC (area under the ROC curve, AUC). Vale ressaltar que o uso de AUCs como alternativa ao uso de ROCs nas análises de desempenho são especialmente úteis quando necessita-se analisar diversos desempenhos muito próximos entre si e/ou que se cruzam em algum ponto do gráfico.

Seja uma rede secundária com $U$ SUs, cada um com uma única antena e coletando $N$ amostras do sinal recebido a cada período de sensoriamento em determinado canal PU. Seja também uma rede primária contendo apenas um transmissor primário. O objetivo do sensoriamento é decidir se o canal PU está livre ou ocupado com base nas amostras de sensoriamento de cada SU a fim de prover acesso oportunista ao espectro. Seja $y_{u}(n)$ a $n$-ésima amostra recebida no $u$-ésimo $\mathrm{SU}$, com $u=1,2, \ldots, U$ e $n=1,2, \ldots, N$. Sob $\mathcal{H}_{0}$ ou $\mathcal{H}_{1}$, tem-se

$$
y_{u}(n)= \begin{cases}\eta_{u}(n) & ; \mathcal{H}_{0} \\ \sum_{z=0}^{Z-1} h_{u}(z) s(n-z)+\eta_{u}(n) & ; \mathcal{H}_{1}\end{cases}
$$

tal que $\eta_{u}(n)$ e $s(n)$ representam a $n$-ésima amostra complexa de ruído aditivo Gaussiano branco (additive white Gaussian noise, AWGN), com média zero e variância $\sigma^{2}$, e a $n$-ésima amostra complexa do sinal (quadrature phase-shift keying, QPSK) emitido pelo transmissor primário, respectivamente. A parcela $\sum_{z=0}^{Z-1} h_{u}(z) s(n-z)$ é o resultado da operação de convolução entre a $n$-ésima amostra do sinal PU e a reposta ao impulso do canal de sensoriamento entre o transmissor primário e o $u$-ésimo SU. A resposta ao impulso é variante no tempo e possui linha de atraso com $Z$ derivações. Com $Z=1, h_{u}(z)$ configura um canal com sombreamento e desvanecimento plano e lento, e com $Z>1$ um canal com sombreamento e desvanecimento lento e seletivo em frequência [5].

\section{A. Canal de sensoriamento}

Os canais de sensoriamento formados entre PU e SUs levam em conta o modelo Rayleigh-lognormal proposto em [8]. Os ganhos dos $Z$ percursos de propagação entre o transmissor primário e o $u$-ésimo SU em (1), $h_{u}(z), z=0,1, \ldots, Z-1$, podem ser escritos como

$$
h_{u}(z)=h_{u}^{\mathrm{f}}(z)+h_{u}^{\mathrm{s}}(z),
$$

sendo $h_{u}^{\mathrm{f}}(z)$ e $h_{u}^{\mathrm{s}}(z)$ representantes dos ganhos de canal referentes ao desvanecimento multipercurso (multipath fading) e sombreamento (shadowing), respectivamente. Os $Z$ ganhos de percurso podem ser organizados em um vetor $\left(\mathbf{h}_{u}^{\mathrm{f}}\right)^{\dagger} \in \mathbb{C}^{Z \times 1}$, em que $\dagger$ representa a operação conjugado transposto. Cada elemento deste vetor possui distribuição Gaussiana complexa independente e identicamente distribuída (i.i.d.), com média zero e variância unitária, i.e., $\mathbb{E}\left[\mathbf{h}_{u}^{\mathrm{f}}\left(\mathbf{h}_{u}^{\mathrm{f}}\right)^{\dagger}\right]=1$, tal que $\mathbb{E}[\cdot]$ representa o operador esperança estatística. Os ganhos são mantidos constantes em um dado período de sensoriamento e variando entre um período e outro, simulando um canal com desvanecimento lento e seletivo em frequência. Já o vetor $\left(\mathbf{h}_{u}^{\mathrm{s}}\right)^{\mathrm{T}} \in \mathbb{C}^{Z \times 1}$, em que $\mathrm{T}$ indica operação de transposição, possui elementos idênticos, cujos módulos têm distribuição normal em escala logarítmica (lognormal) e fase com distribuição uniforme em $[0,2 \pi)$. Ressalta-se que estes elementos são idênticos porque representam o sombreamento sobre todos os ganhos de percurso do canal entre o transmissor primário e o $u$-ésimo SU. 
Os SUs operam sob canais com sombreamento espacialmente correlacionado segundo o modelo tridimensional (3D) proposto em [8]. Esse modelo é baseado em grid points, como mostra a Figura 1, na qual o espaço tridimensional, com $K \times K \times K$ metros cúbicos, é a região de operação dos SUs. Os grid points determinam os pontos onde a correlação é nula. Assim, SUs localizados exatamente em diferentes grid points estarão operando sob canais totalmente descorrelacionados. Por isso, a distância entre grid points adjacentes é chamada de distância de descorrelação (decorrelation distance, $D_{\mathrm{dec}}$ ).

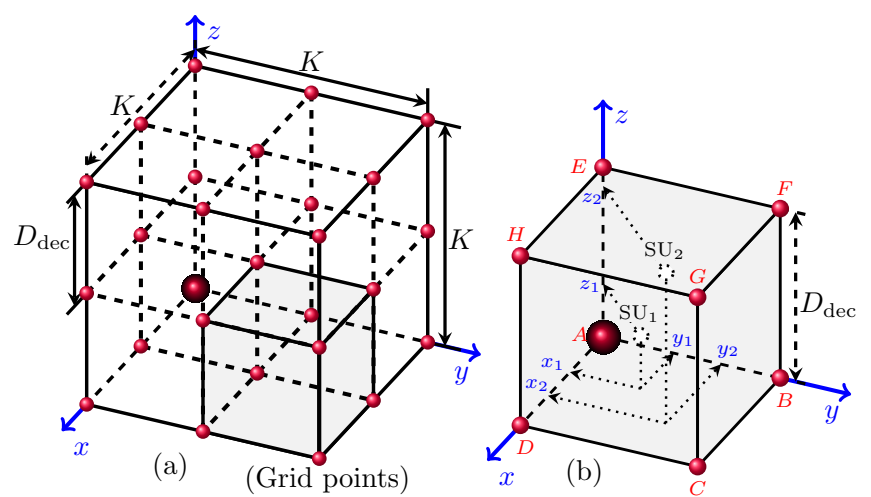

Fig. 1: Ilustração do modelo 3D de canal com sombreamento espacialmente correlacionado.

A Figura 1(b) mostra uma versão ampliada de um dos cubos da Figura 1(a). Por meio dela, nota-se que o nível de correlação do sombreamento aumenta com o aumento de $D_{\mathrm{dec}}$ se os deslocamentos que separam dois ou mais SUs forem menores que o aumento de $D_{\mathrm{dec}}$. O oposto ocorre com a redução de $D_{\text {dec }}$ se os deslocamentos que separam dois ou mais SUs forem menores que esta redução. A posição do $u$ ésimo SU é determinada pelas coordenadas $x_{u}, y_{u}$ e $z_{u}$ em relação a um ponto específico do cubo em que se encontra. Na Figura 1(b) este ponto específico é o grid point $A$. No exemplo da Figura 1(b), os sombreamentos que afetam os sinais recebidos nos SUs 1 e 2 possuem correlação não nula, já que ambos se encontram dentro do mesmo conjunto de grid points e suas coordenadas são inferiores as destes grid points. Cada grid point representa uma amostra Gaussiana de média zero e desvio padrão $\sigma_{\mathrm{dB}}$, sendo esse o desvio padrão do sombreamento. Os pontos $A, B, C, D, E, F, G$ e $H$, portanto, representam amostras Gaussianas dadas por $S_{\mathrm{a}}, S_{\mathrm{b}}$, $S_{\mathrm{c}}, S_{\mathrm{d}}, S_{\mathrm{e}}, S_{\mathrm{f}}, S_{\mathrm{g}}$ e $S_{\mathrm{h}}$, respectivamente.

A correlação espacial do sombreamento que afeta o $u$ ésimo SU é produzida por meio de uma combinação linear associada aos 8 grid points aos quais pertence esse SU. Por exemplo, dados os grid points de $A$ a $H$ da Figura 1(b), uma amostra de sombreamento para o $u$-ésimo SU é obtida como $S_{u}=\left[\left(A \tilde{x}_{u}^{\prime}+B \tilde{x}_{u}\right) \tilde{y}_{u}^{\prime}+\left(C \tilde{x}_{u}^{\prime}+D \tilde{x}_{u}\right) \tilde{y}_{u}\right] \tilde{z}_{u}^{\prime}+$ $\left[\left(E \tilde{x}_{u}^{\prime}+F \tilde{x}_{u}\right) \tilde{y}_{u}^{\prime}+\left(G \tilde{x}_{u}^{\prime}+H \tilde{x}_{u}\right) \tilde{y}_{u}\right] \tilde{z}_{u}$, em que $\tilde{x}_{u}=x_{u} / D_{\mathrm{dec}}$, $\tilde{y}_{u}=y_{u} / D_{\operatorname{dec}}$ e $\tilde{z}_{u}=z_{u} / D_{\operatorname{dec}}$ são distâncias normalizadas em relação ao grid point de referência (grid point $A$ ), e $\tilde{x}_{u}^{\prime}=1-\tilde{x}_{u}, \tilde{y}_{u}^{\prime}=1-\tilde{y}_{u}$ e $\tilde{z}_{u}^{\prime}=1-\tilde{z}_{u}$. Para que o desvio padrão de $S_{u}$ seja igual a $\sigma_{\mathrm{dB}}$, deve-se fazer a correção

$$
S_{u}^{\prime}=\frac{S_{u}}{\sqrt{\left(1-2 \tilde{x}_{u}+2 \tilde{x}_{u}^{2}\right)\left(1-2 \tilde{y}_{u}+2 \tilde{y}_{u}^{2}\right)\left(1-2 \tilde{z}_{u}+2 \tilde{z}_{u}^{2}\right)}}
$$

sendo $S_{u}^{\prime}$ a variável Gaussiana final que representa a amostra de sombreamento que afeta o sinal recebido no $u$-ésimo SU.

Nota-se que a correlação entre as amostras de sombreamento geradas pelo modelo apresentado são dependentes das coordenadas dos SUs e do valor de $D_{\text {dec }}$. Vale ressaltar que com $z_{u}=0$ este modelo $3 \mathrm{D}$ se converte no modelo bidimensional (2D) apresentado em [8].

\section{B. GLRT}

O GLRT é um teste estatístico aplicado a partir do cálculo dos autovalores da matriz de covariância, R, das amostras dos sinal recebido. No sensoriamento espectral cooperativo centralizado com fusão de dados, $\mathbf{R}$ é computada no FC a partir da matriz de amostras recebidas, $\mathbf{Y}$, que contém as amostras enviadas por todos os SUs em cooperação. Então, sejam as $U N$ amostras complexas recebidas no FC organizadas na matriz $\mathbf{Y} \in \mathbb{C}^{U \times N}$, tal que $\mathbf{Y}=\left[\mathbf{y}_{1}, \mathbf{y}_{2}, \cdots, \mathbf{y}_{U}\right]^{\mathrm{T}}$, sendo $\mathbf{y}_{u}=\left[y_{u}(1), y_{u}(2), \ldots, y_{u}(N)\right]^{\mathrm{T}}$ um vetor contendo as $N$ amostras coletadas pelo $u$-ésimo $\mathrm{SU}$ (vide Eq. (1)). A matriz de covariância amostral é dada por $\mathbf{R}=\mathbf{Y Y}^{\dagger} / N$, $\mathbf{R} \in \mathbb{C}^{U \times U}$. A estatística de teste do GLRT pode ser então calculada como

$$
T_{\mathrm{GLRT}}=\frac{\lambda_{1}}{\frac{1}{U} \sum_{u=1}^{U} \lambda_{u}},
$$

tal que $\lambda_{1} \geq \lambda_{2} \geq \cdots \geq \lambda_{U-1} \geq \lambda_{U}$ são os autovalores da matriz $\mathbf{R}$ organizados em ordem decrescente. Ou seja, a estatística de teste do GLRT é computada pela razão entre o máximo autovalor e a média dos autovalores da matriz de covariância amostral R. A decisão de ocupação é obtida comparando-se $T_{\mathrm{GLRT}}$ com um limiar de decisão predefinido, $\gamma$. Decide-se em favor de $\mathcal{H}_{1}$ se $T_{\mathrm{GLRT}}>\gamma$ e em favor de $\mathcal{H}_{0}$ caso contrário.

\section{CFCPSC}

O CFCPSC [5] é um algoritmo baseado na estimativa da PSD, ou periodograma, das amostras do sinal recebido durante o sensoriamento realizado por cada SU. A título de ilustração, a Figura 2 mostra uma PSD hipotética teórica de um sinal QPSK, representativa de uma PSD do sinal transmitido por determinado PU, e esta mesma PSD somada com um certo nível de potência de ruído na recepção a fim de representar a PSD do sinal recebido em um dado SU.

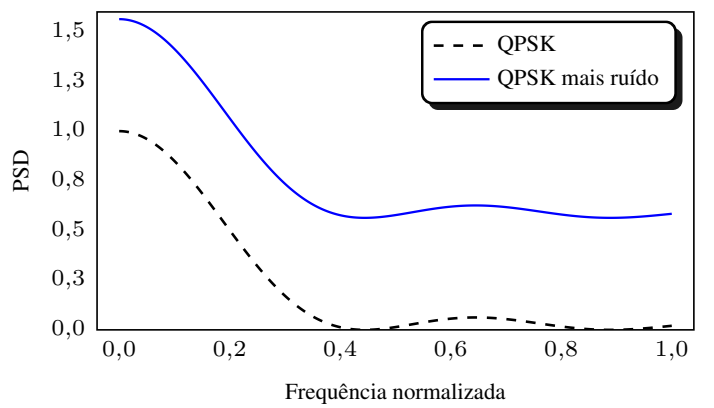

Fig. 2: PSDs hipotéticas do sinal PU (QPSK) e do sinal recebido nos SUs (QPSK mais ruído).

No algoritmo CFCPSC a PSD, estimada via amostras do $u$ ésimo SU, é dividida em $L$ sub-bandas e, após a combinação 
dos dados de todos os SUs, uma decisão sobre a presença do sinal PU é tomada em relação à $\ell$-ésima sub-banda, com $\ell=1,2, \ldots, L$. Esta decisão é obtida por meio do cálculo da $\ell$-ésima estatística de teste, dada por $r_{\ell}^{\text {avg }}$. A decisão final de ocupação do canal PU sensoriado é tomada após a combinação das decisões individuais em relação a cada sub-banda. O maior poder estatístico do CFCPSC, portanto, é proporcional à maior concentração da PSD nas sub-bandas localizadas em baixas frequências. Em outras palavras, as decisões sobre a presença do sinal PU nestas sub-bandas são mais precisas, quando comparadas às decisões tomadas em sub-bandas localizadas em altas frequências, melhorando o desempenho da decisão final. Isso porque essas sub-bandas localizam-se em regiões de maior relação sinal-ruído (signal-to-noise ratio, $\mathrm{SNR}$ ) em relação às sub-bandas localizadas mais à direita da PSD (Imaginar a divisão da PSD da Figura 2 em $L$ sub-bandas de mesmo tamanho pode ser útil para identificar as regiões de maior SNR). Os passos a seguir podem ser usados para descrever o algoritmo CFCPSC no FC:

1) Estime a PSD de $y_{u}(n)$ em (1) via transformada discreta de Fourier (discrete Fourier transform, DFT) como $F_{u}^{\prime}(n)=\frac{1}{N}\left|\operatorname{DFT}\left\{y_{u}(n)\right\}\right|^{2}$

2) Realize o dobramento circular de $F_{u}^{\prime}(n)$ como $F_{u}(n)=$ $\left(F_{u}^{\prime}(1)+F_{u}^{\prime}(N / 2+1)\right) / 2$ para $n=1$, e como $F_{u}(n)=$ $\left(F_{u}^{\prime}(n)+F_{u}^{\prime}(N-n+2)\right) / 2$ para $n=2,3, \ldots, N$;

3) Divida $F_{u}(n)$ em $L$ sub-bandas, com $V=N /(2 L)$ amostras cada, e então calcule a quantidade $F_{u, \ell}=$ $\sum_{n=1}^{V} F_{u}((\ell-1) V+n)$ na $\ell$-ésima sub-banda, $\ell=$ $1,2, \ldots, L$;

4) Calcule a energia total do sinal proveniente do $u$-ésimo SU via $F_{u}^{\text {full }}=\sum_{n=1}^{N / 2} F_{u}(n)$;

5) Calcule $r_{u, \ell}=F_{u, \ell} / F_{u}^{\text {full }}$ para a $\ell$-ésima sub-banda referente ao $u$-ésimo $\mathrm{SU}$;

6) Calcule a média de $r_{u, \ell}$ em relação a todos os SUs na $\ell$-ésima sub-banda, resultando nas estatísticas de teste $r_{\ell}^{\mathrm{avg}}=\frac{1}{U} \sum_{u=1}^{U} r_{u, \ell}$

7) Compare $r_{\ell}^{\text {avg }}$ com um limiar de decisão $\gamma$ predefinido e tome a decisão de ocupação em relação à $\ell$-ésima subbanda como $\mathcal{H}_{0}$ se $r_{\ell}^{\text {avg }}<\gamma$, ou $\mathcal{H}_{1}$ se $r_{\ell}^{\text {avg }}>\gamma$;

8) Tome a decisão de ocupação do canal PU como $\mathcal{H}_{0}$ se todas as decisões por sub-banda forem em favor de $\mathcal{H}_{0}$; ou $\mathcal{H}_{1}$ se pelo menos uma das decisões por sub-banda for em favor de $\mathcal{H}_{1}$.

\section{WCFCPSC}

O WCFCPSC [7] é a versão ponderada do algoritmo CFCPSC que possui maior poder estatístico de detecção. A única diferença entre eles é a proposta para o esquema de ponderação que concede maior peso às estatísticas de teste das sub-bandas localizadas nas regiões de maior SNR da PSD do sinal recebido (vide Figura 2, curva "QPSK mais ruído"). Consequentemente, o algoritmo WCFCPSC é computado com os mesmos Passos 1,2,3,4,5 e 8 do CFCPSC. O Passo 6, porém, é modificado a fim de que a estatística de teste da $\ell$-ésima sub-banda, $r_{\ell}^{\text {avg }}$, seja ponderada pelo peso

$$
w_{\ell}=\frac{L-\ell+1}{L} .
$$

A $\ell$-ésima estatística de teste ponderada é então dada por

$$
r_{\ell}^{\mathrm{avg}-\mathrm{w}}=w_{\ell} r_{\ell}^{\mathrm{avg}},
$$

em que o fator $1 / U$, usado no cálculo de $r_{\ell}^{\text {avg }}$, pode ser suprimido ou incorporado em $w_{\ell}$, já que esta constante não altera o desempenho. No Passo 7, substitui-se $r_{\ell}^{\text {avg }}$ por $r_{\ell}^{\text {avg-w }}$.

Nota-se que (5) caracteriza-se por um esquema de pesos com decaimento linear à medida que o valor de $\ell$ aumenta. Por exemplo, se $L=5$, então $w_{\ell}=[1 ; 0,8 ; 0,6 ; 0,4 ; 0,2]$ para $\ell=1,2, \ldots, 5$, respectivamente. Por isso, considerando que o sinal primário é determinístico, QPSK, e portanto possui PSD com formato semelhante ao mostrado na Figura 2, então (6) favorece os valores de $r_{\ell}^{\text {avg }}$ correspondentes às sub-bandas com maior SNR, enquanto penaliza os valores correspondentes à cauda da PSD do sinal recebido, região em que a SNR do sinal recebido é menor. Por este motivo o WCFCPSC possui maior poder estatístico de detecção que o CFCPSC.

\section{RESUlTAdOS NumÉRICOS}

Esta seção apresenta os desempenhos do GLRT, CFCPSC e WCFCPSC nos mesmos cenários considerados nas análises de desempenhos do CFCPSC e GLRT em [5]. As simulações foram feitas em Matlab com 50.000 eventos de Monte Carlo. Adotou-se $U=6$ SUs e as amostras do sinal recebido em cada SU a cada período de sensoriamento foram enviadas ao FC, para combinação, via canal de controle perfeito (livre de erros). Sob ruído uniforme, considerou-se $\sigma_{u}^{2}=1, \forall u$, sendo $\sigma_{u}^{2}$ a potência de ruído no $u$-ésimo $\mathrm{SU}$. Sob ruído não uniforme: $\left[\sigma_{1}^{2} ; \sigma_{2}^{2} ; \cdots ; \sigma_{6}^{2}\right]=[0,8 ; 0,9 ; 0,95 ; 1,1 ; 0,85 ; 1,15]$. O sinal PU, QPSK, foi gerado com amostras i.i.d. a cada período de sensoriamento, com 4 amostras por símbolo, e 40 símbolos, resultando em $N=160$ amostras, e sua potência foi ajustada como $\frac{10^{\mathrm{SNR} / 10}}{U} \sum_{u=1}^{U} \sigma_{u}^{2}$ a fim de manter a SNR média desejada para a rede secundária, que foi de $-10 \mathrm{~dB}$. Adotouse $L=5$ sub-bandas para ambos, CFCPSC e WCFCPSC. A área de operação dos SUs, com $K \times K \times K$ metros cúbicos, foi estabelecida com $K=60$ e as coordenadas dos SUs, $x_{u}, y_{u}$ e $z_{u}$, variaram uniformemente entre $[0, K]$ metros a cada período de sensoriamento. Utilizou-se um processo de sombreamento i.i.d. entre um período de sensoriamento e outro, com desvio padrão $\sigma_{\mathrm{dB}}=4$ e distância de descorrelação de $D_{\mathrm{dec}}=30$ metros. Os $Z$ ganhos de percursos de cada canal de sensoriamento são i.i.d.. Com $Z=1$, o canal PUSU é plano e lento, com $Z=4$, seletivo em frequência. Adotou-se ainda canais com resposta ao impulso seguindo o formato típico exponencial com ganhos médios iguais a $[1 ; 0,464 ; 0,215 ; 0,1]$.

A Figura 3 mostra as ROCs/AUCs obtidas pelo WCFCPSC, CFCPSC e GLRT nos 4 cenários adotados em [5]. Estes cenários são formados de acordo com as configurações dos canais de sensoriamento e do ruído na recepção conforme mostra a Tabela I.

Primeiramente, em [5], nos Cenários 1 e 2, ou seja, SUs operando sob ruído uniforme e/ou sob canais seletivos, o GLRT superou significativamente o CFCPSC. Estes resultados também podem ser notados nos gráficos (a) e (b) da Figura 3, respectivamente. Já no Cenário 3 , SUs sob ruído uniforme, canais seletivos e com sombreamento correlacionado, o CFCPSC 
obteve desempenhos ligeiramente superiores ao GLRT, como também pode ser visto no gráfico (c) da Figura 3. Por fim, no Cenário 4, SUs sob canais seletivos, com sombreamento correlacionado e sob ruído não uniforme, o CFCPSC superou consideravelmente o GLRT em [5]. De igual modo, o melhor desempenho do CFCPSC neste cenário também pode ser visto no gráfico (d) da Figura 3. Veja, no entanto, que o WCFCPSC superou o CFCPSC em todos os cenários, bem como superou o GLRT nos cenários em que este havia superado o CFCPSC. Observando as degradações de desempenhos provocadas pela mudança dos Cenários de 1 ao 4 , nota-se que o GLRT possui maior sensibilidade a canais seletivos, com sombreamento correlacionado e, especialmente, sob ruído não uniforme. A maior robustez do CFCPSC e WCFCPSC nestes cenários é facilmente percebida, bem como a superioridade do WCFCPSC em todos eles.
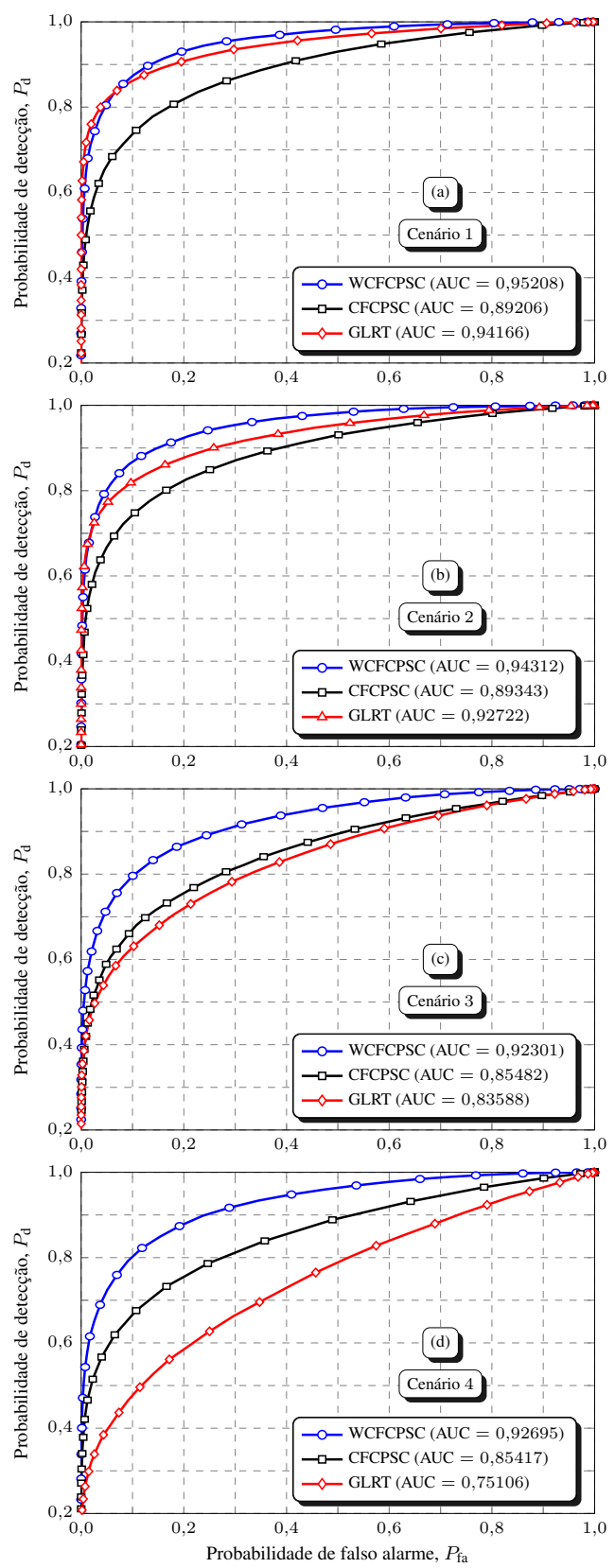

Fig. 3: ROCs WCFCPSC, CFCPSC e GLRT nos Cenários $1,2,3$ e 4.
TABELA I: Cenários de sensoriamento adotados neste artigo e em [5].

\begin{tabular}{|c|c|c|c|}
\hline \hline Cenário & Ruído & $Z$ & Sombreamento \\
\hline 1 & uniforme & 1 & - \\
\hline 2 & uniforme & 4 & - \\
\hline 3 & uniforme & 4 & $\checkmark$ \\
\hline 4 & não uniforme & 4 & $\checkmark$ \\
\hline \hline
\end{tabular}

\section{CONCLUSÕES}

Neste artigo analisou-se os desempenhos de três estatísticas de teste no sensoriamento espectral cooperativo e centralizado, com fusão de dados, sob ruído não uniforme e canais seletivos em frequência e com sombreamento espacialmente correlacionado. Os desempenhos do WCFCPSC e CFCPSC foram comparados com os desempenhos obtidos pelo bem conhecido teste GLRT e mostram o melhor desempenho do WCFCPSC até mesmo nos casos em que a versão original CFCPSC obteve desempenhos inferiores aos obtidos pelo GLRT. Além disso, os desempenhos do GLRT mostraram-se consideravelmente mais sensíveis a ruído não uniforme. As análises levaram em conta os cenários adotados na publicação do CFCPSC original a fim de destacar a superioridade do WCFCPSC sobre ambos, GLRT e CFCPSC. Ressalta-se, no entanto, a relevância de se estender as análises a outros cenários. Por exemplo, poderse-ia adotar outros modelos de canal de sensoriamento, como o modelo 3D desenvolvido pelo third generation partnership project (3GPP), que leva em conta perda de percurso (pathloss) e probabilidade de linha de visada (line-of-sight, LoS) e/ou canal de controle imperfeito, bem como modelos de seletividade em frequência também mais realistas. Por fim, como uma extensão deste trabalho, pretende-se comparar os resultados com os que seriam obtidos por alguma técnica de aprendizado de máquina, já que o uso da inteligência artificial tem sido visto como uma boa alternativa às técnicas convencionais de detecção nos últimos anos [9].

\section{REFERÊNCIAS}

[1] L. Chettri and R. Bera, "A comprehensive survey on Internet of things (IoT) toward 5G wireless systems," IEEE Internet Things J., vol. 7, no. 1, pp. 16-32, 2020.

[2] J. M. C. Brito, L. L. Mendes, and J. G. S. Gontijo, "Brazil 6G project - an approach to build a national-wise framework for $6 \mathrm{G}$ networks," in 2020 2nd 6G Wireless Summit (6G SUMMIT), 2020, pp. 1-5.

[3] M. A. McHenry, P. A. Tenhula, D. McCloskey, D. A. Roberson, and C. S. Hood, "Chicago spectrum occupancy measurements \& analysis and a long-term studies proposal," in Proc. of the first int. workshop on Technology and policy for accessing spectrum. ACM, 2006, p. 1.

[4] B. Nadler, F. Penna, and R. Garello, "Performance of eigenvalue-based signal detectors with known and unknown noise level," in 2011 IEEE Int. Conf. on Commun. (ICC), 2011, pp. 1-5.

[5] R. C. D. V. Bomfin, R. A. A. de Souza, and D. A. Guimarães, "Circular folding cooperative power spectral density split cancellation algorithm for spectrum sensing," IEEE Commun. Lett., vol. 21, no. 2, pp. 250-253, 2017.

[6] L. S. Costa and R. A. A. de Souza, "Performance of blind cooperative spectrum sensing under impulsive noise," in 2020 IEEE Latin-American Conf. on Commun. (LATINCOM), 2020, pp. 1-6.

[7] L. S. Costa, D. A. Guimarães, E. P. Frigieri, and R. A. A. de Souza, "Weighted circular folding cooperative power spectral density split cancellation algorithm," IEEE Trans. Veh. Technol., vol. 70, no. 1, pp. 10621066, 2021

[8] R. C. D. V. Bomfin and R. A. A. de Souza, "A new spatially correlated shadowed channel model with cognitive radio application," in 2015 IEEE 81st Veh. Technol. Conf. (VTC Spring), 2015, pp. 1-5.

[9] S. Solanki, V. Dehalwar, and J. Choudhary, "Deep learning for spectrum sensing in cognitive radio," Symmetry, vol. 13, no. 1, 2021. [Online]. Available: https://www.mdpi.com/2073-8994/13/1/147 